\title{
Kaempferol inhibits interleukin-1 $\beta$ stimulated matrix metalloproteinases by suppressing the MAPK-associated ERK and P38 signaling pathways
}

\author{
XIAOJIAN HUANG, QIYONG PAN, ZEKAI MAO, PENGCHENG WANG, \\ RUI ZHANG, XIAOHU MA, JINGYUAN CHEN and HONGBO YOU \\ Department of Orthopedics, Tongji Hospital, Tongji Medical College, \\ Huazhong University of Science and Technology, Wuhan, Hubei 430030, P.R. China
}

Received October 10, 2017; Accepted June 28, 2018

DOI: $10.3892 / \mathrm{mmr} .2018 .9280$

\begin{abstract}
Osteoarthritis (OA) is a common degenerative joint disease in older adults. A number of previous studies have demonstrated that natural flavonoids can serve as promising therapeutic drugs for OA. Kaempferol, a phytochemical ingredient mainly present in various fruits, has exhibited its prominent anti-inflammatory and antioxidant effects in numerous diseases. However, whether Kaempferol ameliorates the deterioration of arthritis remains to be elucidated. The aim of the present study was to investigate the therapeutic role of Kaempferol on OA in rat chondrocytes. The results revealed that Kaempferol significantly inhibited the interleukin (IL)-1 $\beta$-induced protein expression of inflammatory mediators such as inducible nitric oxide synthase and cyclo-oxygenase-2. In addition, the common matrix degrading enzymes [matrix metalloproteinase (MMP)-1, MMP-3, MMP-13 and a disintegrin and metalloproteinase with thrombospondin motif-5] induced by IL- $1 \beta$ were also suppressed by Kaempferol, and consequently abolished the degradation of collagen II. Furthermore, the anti-inflammatory effect of Kaempferol was mediated by the inhibition of the mitogen activated protein kinase-associated extracellular signal-regulated kinase and P38 signaling pathways. These results collectively indicated that Kaempferol can potentially prevent OA development and serve as a novel pharmacological target in the treatment of OA.
\end{abstract}

Correspondence to: Professor Hongbo You, Department of Orthopedics, Tongji Hospital, Tongji Medical College, Huazhong University of Science and Technology, 1095 Jiefang Avenue, Qiaokou, Wuhan, Hubei 430030, P.R. China

E-mail: hbyou@aliyun.com

Key words: kaempferol, mitogen activated protein kinase, matrix metalloproteinases, a disintegrin and metalloproteinase withthrombospondin motif-5, collagen II

\section{Introduction}

Osteoarthritis (OA) is a common degenerative joint disease in older adults (1). Joint pain, stiffness, motion limitations are the most common symptoms of $\mathrm{OA}$ and it is one of the leading causes of disability worldwide $(2,3)$. Recently one comparative analysis confirms that the disease has doubled in prevalence since the mid-20th century (4). To date, however, there are no modifying agents to halt or reverse the progression of OA. The outcomes of most patients with advanced stage of OA are joint replacement. The surgery, however, costs much and causes great social and economic problems (5).

Disintegration of collagen II in cartilage matrix is regarded as the central pathology of OA and it is commonly accepted that inflammation plays a crucial role in the development of OA $(6,7)$. Overproduction of pro-inflammatory cytokines like Interleukin (IL)-1 $\beta$ can lead to increased secretion of catabolic enzymes such as matrix metalloproteinases (MMPs) and disintegrin and metalloproteinase with thrombospondin motif-5 (ADAMTS-5) in OA cartilage, which represent the major proteolytic enzymes in the degradation of cartilage $(8,9)$. In addition, IL-1 $\beta$ can also increase the expression of other catabolic factors, including Inducible NOS (iNOS) and Cyclo-oxygenase-2 (COX-2), in chondrocytes $(10,11)$. To this end, we primarily utilize rat chondrocytes treated with IL-1 $\beta$ as our fundamental model of OA. in vitro.

Numerous studies have confirmed the anti-inflammatory effects of different flavonoids one the treat of $\mathrm{OA}$ in vitro or in vivo, indicating that flavonoids can represent good therapeutic method for OA (12). Kaempferol (3, 4', 5, 7-tetrahydoxyflavone) is a natural flavonoid commonly found in a variety of foods such as tea, apples, strawberries, beans, and citrus fruits (13). It has been widely concerned for its antitumor, antioxidative, antibacterial and anti-inflammatory properties (14). One clinical study recently found that Elaeagnus Angustifolia (EA) extract containing $0.21 \%$ (w/w) Kaempferol could significantly reduce the symptoms of OA, but its' internal anti-inflammatory effect on chondrocytes remains to be fully elucidated (15). The object of this study is to explore its anti-inflammatory effects on IL-1 $\beta$-induced rat chondrocytes and the internal mechanism. 


\section{Materials and methods}

Chemicals and reagents. Kaempferol, collagenase, dimethyl sulfoxide (DMSO) were purchased from Sigma-Aldrich (Merck KGaA; Darmstadt, Germany). Fetal bovine serum (FBS) was obtained from Glico-BRL (Gaithersburg, MD, USA). Recombinant rat IL-1 $\beta$ was obtained from R\&D systems (Minneapolis, MN, USA). Primary antibodies specific for MMP-3, COX-2, phosphorylated extracellular signal-regulated kinase (p-ERK), ERK, c-Jun N-terminal kinase (JNK), p-JNK, p38, p-p38 were obtained from Cell Signaling Technology Inc. (Beverly, MA, USA). Antibodies specific for iNOS, MMP13, Collagen II was purchased from Santa Cruz Biotechnology, Inc. (Santa Cruz, CA, USA). Anti-MMP1 was obtained from ProteinTech Group (Wuhan, China). Antibodies against glyceraldehyde-3-phosphate dehydrogenase (GAPDH), ADAMTS-5, Secondary antibodies and the cell counting kit-8 (CCK-8) were provided from Wuhan Boster Biological Technology, Ltd. (Wuhan, China).

Rat chondrocytes culture. Animal experiment procedures were approved by the Animal Care and Use Committee of Tongji Medical College (Wuhan, China). 5-day-old Sprague-Dawley rats were used as previously described (16). Briefly, rats were sacrificed by cervical dislocation and articular cartilage was isolated from the knee joints of rats and then cut into pieces. Cartilages pieces were digested in $0.25 \%$ trypsin for $30 \mathrm{~min}$ and then incubated with $0.25 \%$ collagenase II for $8 \mathrm{~h}$ at $37^{\circ} \mathrm{C}$. Chondrocytes were collected and then cultured in complete culture medium supplemented with $10 \%$ FBS, penicillin $(100 \mathrm{IU} / \mathrm{ml})$ and streptomycin $(100 \mu \mathrm{g} / \mathrm{ml})$ at $37^{\circ} \mathrm{C}$ in a humidified atmosphere containing $5 \% \mathrm{CO}_{2}$. Two to three passages were used for our experiment.

Cell viability assay. Cell viability was estimated by the CCK- 8 assay. Briefly, rat chondrocytes were seeded in a 96-well plate ( $2 \times 10^{4} /$ well). After $24 \mathrm{~h}$ of cell adherence, chondrocytes were treated with different concentrations of Kaempferol $(0,5$, 10 and $20 \mu \mathrm{M}$ ) for $24 \mathrm{~h}$. Each well was incubated with $100 \mu \mathrm{l}$ culture medium containing $10 \mu \mathrm{l}$ CCK-8 solution for $1 \mathrm{~h}$ at $37^{\circ} \mathrm{C}$ with $5 \% \mathrm{CO}_{2}$, and then the absorbance was detected at $450 \mathrm{~nm}$ using a micro plate reader (Bio-Rad, Richmond, CA, USA).

$R N A$ isolation and reverse transcription-quantitative polymerase chain reaction $(R T-q P C R)$ analyses. Following the respective treatments, total RNA in each group was extracted using TRIzol (Invitrogen; Thermo Fisher Scientific, Inc., Waltham, MA, USA) according to the manufacturer's protocol. An NanoDrop spectrometer (Thermo Fisher Scientific, Inc.) was used here to detect the concentration and purity of the total RNA. RNA samples were then reverse-transcribed to cDNA via RT-PCR using a ReverTra Ace qPCR RT kit (Toyobo Life Science, Osaka, Japan), as per manufacturer's instructions. The expression of mRNA was determined quantitatively using SYBR-Green Realtime PCR Master Mix (Toyobo Life Science) with the following thermocycling conditions: $95^{\circ} \mathrm{C}$ for $30 \mathrm{sec}$, followed by 40 cycles of $95^{\circ} \mathrm{C}$ for $5 \mathrm{sec}$ and $60^{\circ} \mathrm{C}$ for $30 \mathrm{sec}$. The GAPDH gene was used as an internal control. Each set of samples included a template-free control and data were analyzed using the $2^{-\Delta \Delta C q}$ method (17). Primers used in this study were as follows: GAPDH forward, 5'-CTCATGACC ACAGTCCATGC and reverse, 5'-TTCAGCTCTGGGATG ACCTT-3'; and MMP13 forward, 5'-AAGATGTGGAGTGCC TGATG-3' and reverse, 5'-CCAGTGTAGGTATAGATGGGA AC-3'.

Western blotting analysis. Chondrocytes were co treated with Kaempferol $(0,5,10$ and $20 \mu \mathrm{M})$ and IL-1 $\beta(10 \mathrm{ng} / \mathrm{ml})$ for $24 \mathrm{~h}$, then cells were lysed with cold RIPA Lysis Buffer supplemented with $1 \%$ protease inhibitor cocktail and $1 \%$ phosphatase inhibitor cocktail (Wuhan Boster Biological Technology, Ltd.). BCA protein assay kit (Wuhan Boster Biological Technology, Ltd.) was used here to detect protein concentration in the lysates. Equal amounts of protein $(25 \mu \mathrm{g})$ were fractionated on $10 \%$ SDS-PAGE gels and transferred to a PVDF membrane (EMD Millipore, Billerica, MA, USA). Membranes were firstly hatched in blocking buffer containing $5 \%$ bovine serum albumin powder in Tris-buffered saline with $0.1 \%$ Tween-20 (TBST) for $1 \mathrm{~h}$, and then incubated overnight with primary antibodies at $4^{\circ} \mathrm{C}$. The primary antibodies are as followed: MMP1 (cat. no. 10371-2-AP; 1:1,500 dilution), MMP-3 (cat. no. 14351; 1:1,000 dilution), MMP13 (cat. no. sc-30073; 1:500 dilution), iNOS (cat. no. sc-7271; 1:500 dilution), COX-2 (cat. no. 12882; 1:1,000 dilution), p-ERK (cat. no. 4370; 1:1,000 dilution), ERK (cat. no. 4695; 1:1,000 dilution), JNK (cat. no. 9258; 1:1,000 dilution), p-JNK (cat. no. $9255 ; 1: 1,000$ dilution), p38 (cat. no. 8690; 1:1,000 dilution), p-p38 (cat. no. 4511; 1:1,000 dilution), Collagen II (cat. no. sc-28887; 1:500 dilution), ADAMTS-5 (cat. no. BA3020; 1:500 dilution), GAPDH (cat. no. BM3876; 1:500 dilution). After wash three times with TBST for $15 \mathrm{~min}$, blots were then incubated with HRP-conjugated secondary antibodies (goat Anti-Rabbit IgG, BA1054; 1:5,000 dilution; and goat Anti-Mouse IgG, BA1050, 1:5,000 dilution) for $1 \mathrm{~h}$ at room temperature. The immunoreactive bands were visualized using an ECL System (Wuhan Boster Biological Technology, Ltd.). GAPDH was used as an internal control. The western blots were repeated three times and representative bands were presented.

Immunofluorescence staining. Chondrocytes were firstly treated with $20 \mu \mathrm{M}$ Kaempferol in the presence of $10 \mathrm{ng} / \mathrm{ml}$ IL-1 $\beta$ for $24 \mathrm{~h}$. Then, cells were fixed with $4 \%$ paraformaldehyde for $15 \mathrm{~min}$. After fully washed with phosphate buffered solution (PBS), the fixed cells were blocked in PBS containing 5\% FBS and $0.3 \%$ Triton X-100 for $1 \mathrm{~h}$ and then incubated overnight at $4^{\circ} \mathrm{C}$ with anti-collagen II antibody (cat. no. sc-28887; 1:300 dilution; Santa Cruz Biotechnology Inc.). Finally, the fixed cells were washed and incubated with CY3-goat anti-rabbit IgG (cat. no. BA1032; 1:100 dilution; Wuhan Boster Biological Technology, Ltd.) and observed under a standard fluorescence microscope.

Statistical analysis. All experiments were performed three times using independent samples. Data were presented as the mean \pm standard deviation. Data were analyzed using GraphPad Prism version 6.00 software (GraphPad Software, Inc., La Jolla, CA, USA). Comparisons of multiple groups were performed using one- and two-way analysis of variance 
A

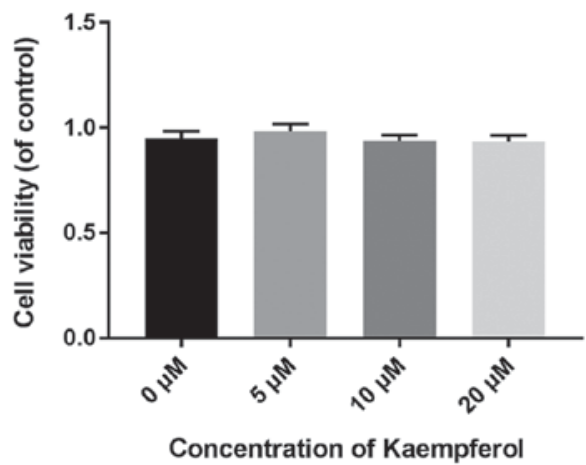

B

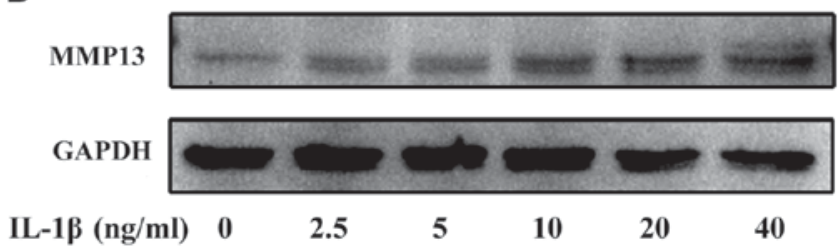

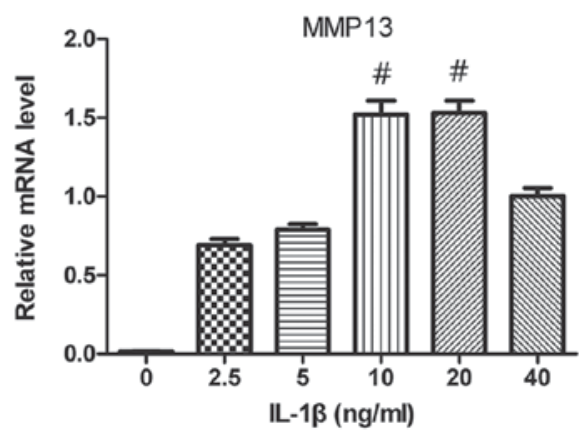

Figure 1. Effects of kaempferol on chondrocyte viability. Cells were treated with IL-1 $\beta(10 \mathrm{ng} / \mathrm{ml})$ in the absence or presence of Kaempferol (5, 10 and $20 \mu \mathrm{M})$ for $24 \mathrm{~h}$. (A) Cell viability was evaluated by Cell Counting kit- 8 assay. The effects of IL-1 $\beta$ on MMP13 expression levels were also evaluated. Chondrocytes were treated with various concentrations of IL- $1 \beta(2.5-40 \mathrm{ng} / \mathrm{ml})$ for $24 \mathrm{~h}$. (B) The protein levels and mRNA expression of MMP13 were detected by western blotting and reverse transcription-quantitative polymerase chain reaction analyses. ${ }^{*} \mathrm{P}<0.05$ vs. control group. IL, interleukin; MMP, matrix metalloproteinase; GAPDH, glyceraldehyde-3-phosphate dehydrogenase.

A

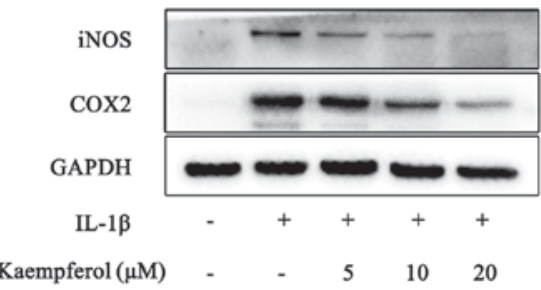

B
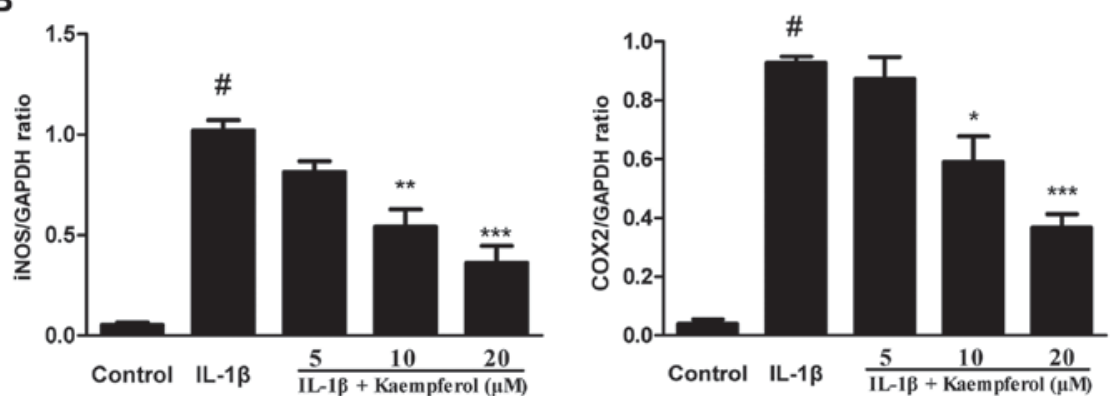

Figure 2. Effects of kaempferol on IL-1 $\beta$-induced PGE2, COX2 and iNOS expression. Cells were treated with IL-1 $\beta$ (10 ng/ml) in the absence or presence of kaempferol $(5,10$ and $20 \mu \mathrm{M})$ for $24 \mathrm{~h}$. (A) The protein levels of iNOS and COX2 were determined by western blot analysis. (B) Relative protein expression was then quantified using Image-J software. The results are representative of three separate experiments. ${ }^{\text {" }} \mathrm{P}<0.05$ vs. control group; ${ }^{*} \mathrm{P}<0.05$, ${ }^{* *} \mathrm{P}<0.01$ and ${ }^{* * *} \mathrm{P}<0.001$ vs. IL-1 $\beta$ group. IL, interleukin; PGE2, prostaglandin E2; COX2, cyclo-oxygenase-2; iNOS, inducible nitric oxide synthase; GAPDH, glyceraldehyde-3-phosphate dehydrogenase.

analysis with a post hoc Tukey's test. $\mathrm{P}<0.05$ was considered to indicate a statistically significant difference.

\section{Results}

Effects of Kaempferol and IL-1 $\beta$ on chondrocytes viability and MMP13 expression. The potential toxicity of Kaempferol on chondrocytes was evaluated by CCK-8 assay. As shown in Fig. 1A, there were no significant difference between the viability of cells in the control and that in the $5-20 \mu \mathrm{M}$ of Kaempferol treatment. To select the appropriate concentration of IL-1 $\beta$ used in vitro model of OA, chondrocytes were treated with various concentrations of IL-1 $\beta(2.5-40 \mathrm{ng} / \mathrm{ml})$ for $24 \mathrm{~h}$. The protein level and mRNA expression of MMP13 were used as an indicator here. As shown in Fig. 1B, the expression level of MMP13 peaks at the $10 \mathrm{ng} / \mathrm{ml}$ of IL-1 $\beta$. These results indicated that $10 \mathrm{ng} / \mathrm{ml}$ was the appropriate concentration of IL-1 $\beta$ for further experiments. 
A

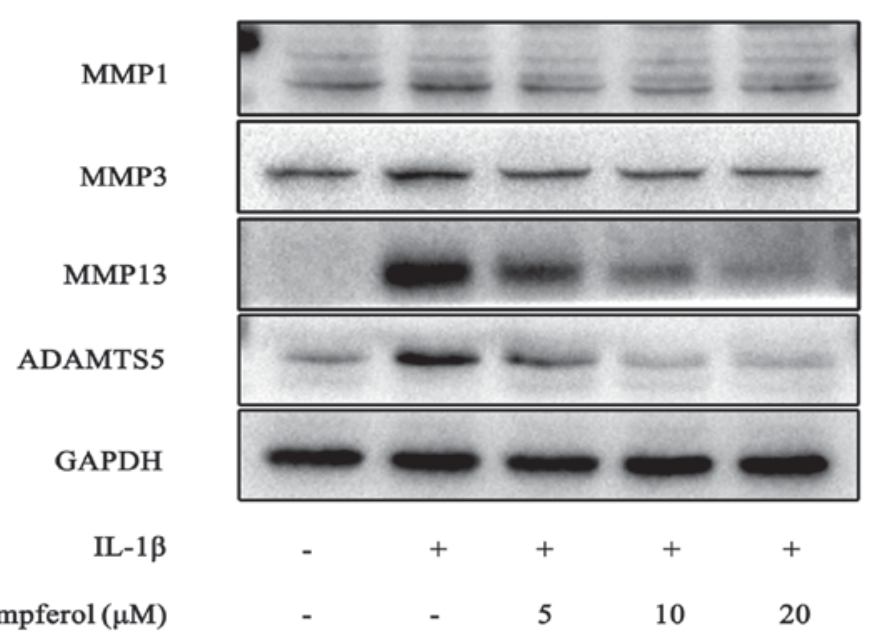

B
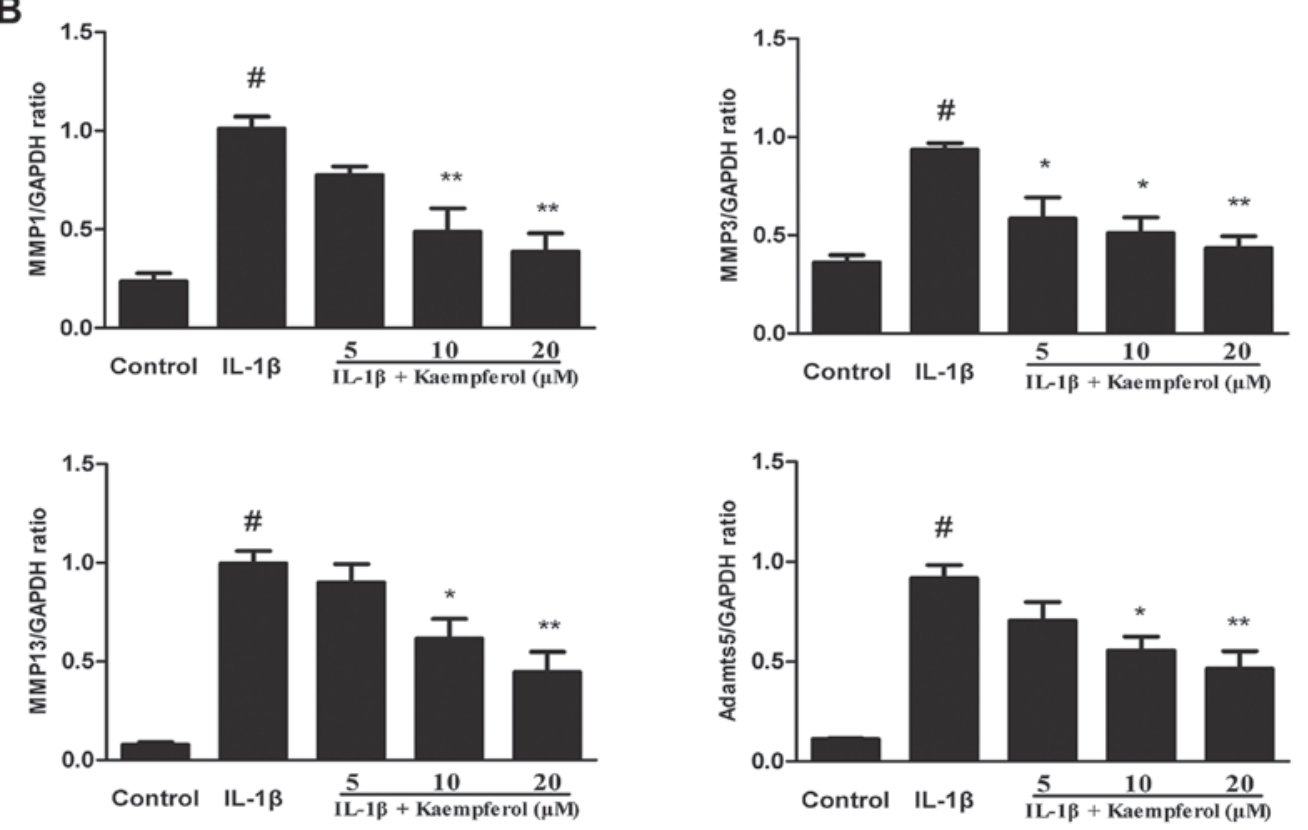

Figure 3. Effects of kaempferol on IL-1 $\beta$-induced MMPs and ADAMTS5 expression. Cells were treated with IL-1 $\beta$ (10 ng/ml) in the absence or presence of kaempferol (5, 10 and $20 \mu \mathrm{M}$ ) for $24 \mathrm{~h}$. (A) The protein levels of MMP1, MMP3, MMP13 and ADAMTS5 were determined by western blot analysis (B) Relative protein expression was then quantified using Image-J software. The results are representative of three separate experiments. ${ }^{\#} \mathrm{P}<0.05 \mathrm{vs}$. control group; " $\mathrm{P}<0.05$ and ${ }^{* *} \mathrm{P}<0.01$ vs. IL-1 $\beta$ group. IL, interleukin; MMP, matrix metalloproteinase; ADAMTS5, a disintegrin and metalloproteinase with thrombospondin motif-5; GAPDH, glyceraldehyde-3-phosphate dehydrogenase.

Effects of Kaempferol on IL-1 $\beta$-induced COX2 and iNOS expression. Pro-inflammatory mediators such as COX2 and iNOS play a key role in the pathogenesis of OA. So we investigated effects of Kaempferol on IL-1 $\beta$-Induced Cox 2 and iNOS expression. The expression of COX2 and iNOS were measured by western blot. As shown in Fig. 2, chondrocytes stimulated with IL-1 $\beta(10 \mathrm{ng} / \mathrm{ml})$ showed enhanced production of COX2 and iNOS. However, chondrocytes treated with Kaempferol can significantly inhibit the IL- $1 \beta$-induced expression of COX2 and iNOS.

Effects of Kaempferol on IL-1 $\beta$-induced MMPs, ADAMTS5 expression. It is widely known that MMPs and ADAMTS5 are the major catabolic enzymes of the cartilage matrix. Therefore, we investigated the effects of Kaempferol on the protein production of MMP-1, MMP-3, MMP-13, and ADAMTS5.
As shown in Fig. 3, IL-1 $\beta$ significantly increased the expression of MMPs and the degradation of collagen II. However, Kaempferol $(5,10$ and $20 \mu \mathrm{M})$ dose-dependently decreased the protein expression levels of MMP1, MMP3, MMP13 and ADAMTS5 induced by IL-1 $\beta$.

Effects of Kaempferol on IL-1 $\beta$-induced collagen II degradation. Collagen II is one of the major components of cartilage matrix. So we explored the effects of Kaempferol on IL-1 $\beta$-Induced collagen II degradation. The expression of collagen II was measured by western blot and Immunohistochemistry. As shown in Fig. 4, IL-1 $\beta$ obviously increased the degradation of collagen II and Kaempferol $(5,10$ and $20 \mu \mathrm{M})$ could suppress this process in protein levels. The fluorescent results also contributed to the same outcome. Compared to the control group, the positive expression areas 
A

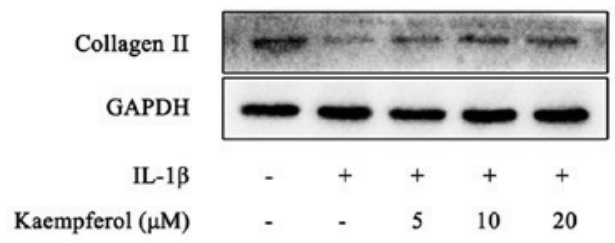

B

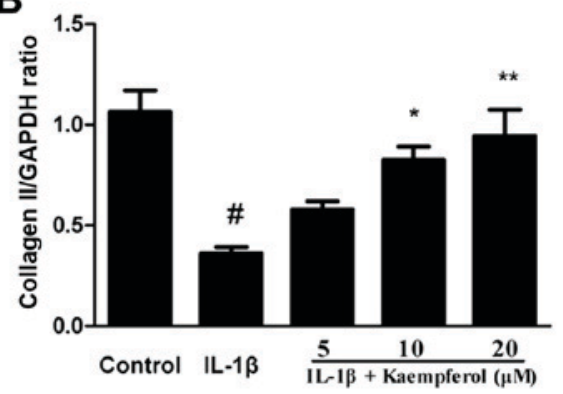

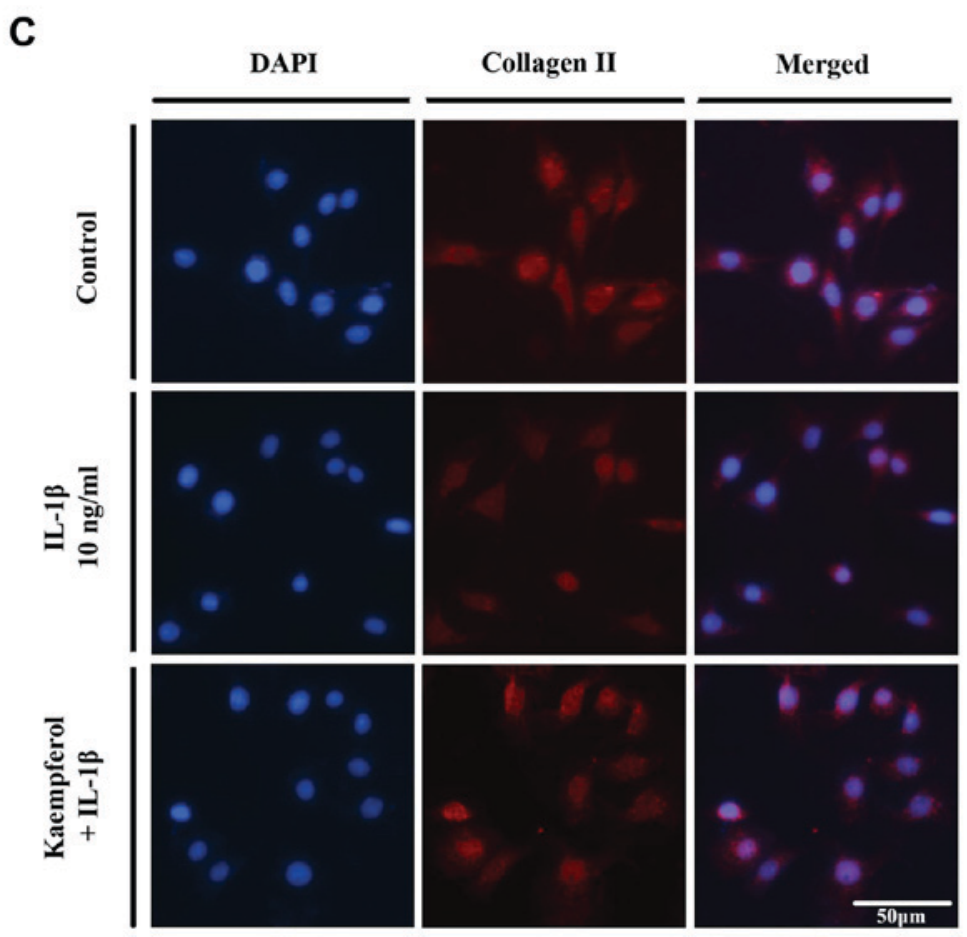

Figure 4. Effects of kaempferol on IL-1 $\beta$-induced collagen II degradation. Cells were treated with IL-1 $\beta(10 \mathrm{ng} / \mathrm{ml})$ in the absence or presence of kaempferol $(5,10$ and $20 \mu \mathrm{M})$ for $24 \mathrm{~h}$. (A) The protein levels of collagen II were detected by western blot analysis. (B) Relative protein expression of collagen II was then quantified using Image-J software. (C) The immunofluorescence results of collagen II in chondrocytes treated with $20 \mu \mathrm{M}$ kaempferol in the presence of $10 \mathrm{ng} / \mathrm{ml} \mathrm{IL}-1 \beta$ for $24 \mathrm{~h}$ (magnification, x200; scale bar, $50 \mu \mathrm{m}$ ). The results are representative of three separate experiments. ${ }^{\# P}<0.05 \mathrm{vs}$. control group; ${ }^{*} \mathrm{P}<0.05$ and ${ }^{* *} \mathrm{P}<0.01$ vs. IL-1 $\beta$ group. IL, interleukin; GAPDH, glyceraldehyde-3-phosphate dehydrogenase.

of collagen II were significantly lower in the IL-1 $\beta$ group. Compared to the IL-1 $\beta$ group, there were significantly increased levels of collagen II in chondrocytes treated with $20 \mu \mathrm{M}$ Kaempferol.

Effects of Kaempferol on mitogen activated protein kinase (MAPK) signaling pathway. MAPK pathways participate in the process of cartilage degradation. To identify the signaling pathway involved in the anti-inflammatory effects of Kaempferol, chondrocytes were co treated with Kaempferol $(0,5,10$ and $20 \mu \mathrm{M})$ and IL- $1 \beta(10 \mathrm{ng} / \mathrm{ml})$ for $30 \mathrm{~min}$. As shown in Fig. 5, IL-1 $\beta$ upregulated the protein level of P-P38, P-ERK, P-JNK, while Kaempferol could downregulated the activation of P38 and ERK. It is interesting that we did not observe significant change of JNK pathway.

\section{Discussion}

OA is conventionally defined as a typical non-inflammatory joint disease, but nowadays it is increasingly accepted that it is an inflammatory disease $(18,19)$. Mounting evidence has demonstrated that inflammation contributes to the symptoms and the progression of OA (20-22). Pro-inflammatory cytokines, such as IL-1 $\beta$, is known to be capable of eliciting inflammatory responses. Various studies have confirmed that IL-1 $\beta$ can block the synthesis of the key structural proteins in chondrocytes such as type-II collagen and aggrecan (23). Besides IL-1 $\beta$ also increases the expression of MMPs and aggrecanases, which are a class of proteinases involved in the cartilage degradation (24). Furthermore, IL-1 $\beta$ can also increase the expression of iNOS and COX-2 in chondrocytes, which inhibit cartilage matrix synthesis and promote its degradation (25). Thus, IL-1 $\beta$ is selected here to develop a cellular OA model and treatment that can reverse IL-1 $\beta$ induced inflammation responses may provide new venues for the therapy for OA. In this study, we certified that Kaempferol inhibited IL- $1 \beta$ induced iNOS and COX- 2 production and the degeneration of collagen II. Meanwhile, Kaempferol could inhibit IL-1 $\beta$-induced MMP-1, MMP-3, MMP-13 and ADAMTS5 expression in rat chondrocytes in vitro. These 
A

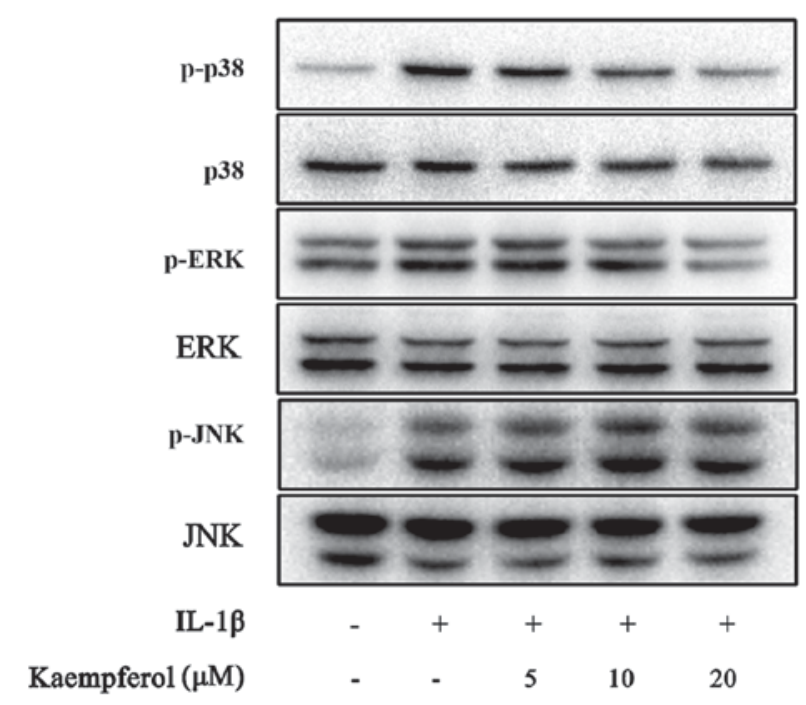

B
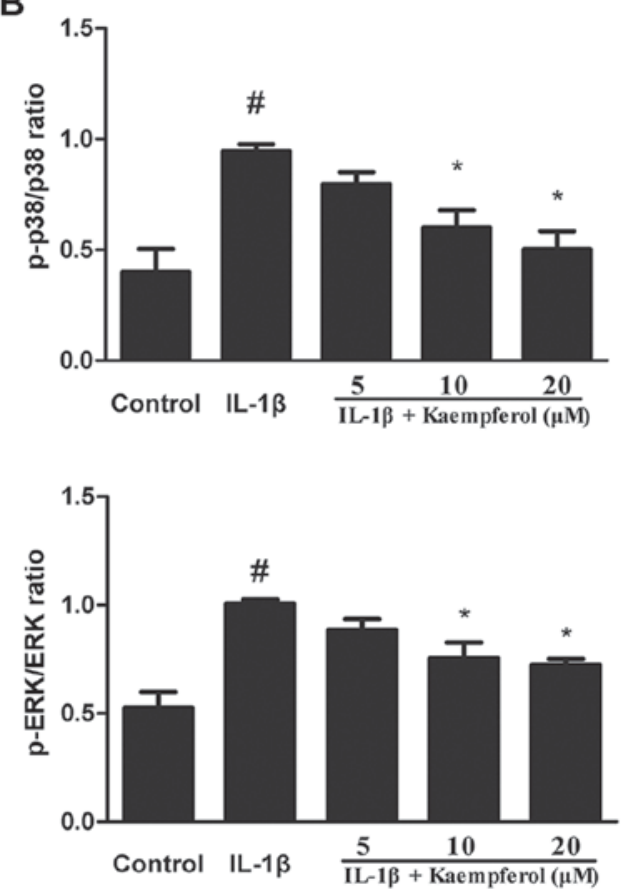

Figure 5. Effects of kaempferol on the MAPK signaling pathway. Cells were treated with IL-1 $(10 \mathrm{ng} / \mathrm{ml})$ in the absence or presence of kaempferol $(5,10$ and $20 \mu \mathrm{M}$ ) for $30 \mathrm{~min}$. (A) The protein levels of P38, ERK and JNK were determined by western blot analysis. (B) Relative protein expression was then quantified using Image-J software. The results are representative of three separate experiments. ${ }^{*} \mathrm{P}<0.05$ vs. control group; ${ }^{\mathrm{P}}<0.05$ vs. IL- $1 \beta$ group. IL, interleukin; MAPK, mitogen activated protein kinase; ERK, extracellular signal-regulated kinase; JNK, c-Jun N-terminal kinase; p-, phosphorylated.

findings collectively demonstrate the anti-inflammatory effects of Kaempferol against OA. However, in this study we failed to elucidate its protective effects in OA in animal experiments. Further research is required to clarify its mechanism in vivo.

MAPK, including JNK, p38, and ERK signaling pathways, is the central node of multiple signal transduction pathways. A large number of experiments suggested that it was a key upstream signaling pathways in the regulation of inflammatory mediators production involved in the pathogenesis of OA (26). Activation of the MAPK signaling pathway may lead to the secretion of several matrix-degrading proteinases, including the MMPs and the aggrecanases, leading to articular cartilage breakdown. IL-1 $\beta$, the major proinflammatory cytokine involved the progression of OA, can easily activated the MAPK pathways $(27,28)$. Thus, in our study, we investigated whether the anti-inflammatory effects of Kaempferol were through the attenuation of MAPK. It was observed that the JNK, p38, ERK signaling pathways were obviously activated upon IL-1 $\beta$ stimulation. However, Kaempferol treatment dose-dependently suppressed the phosphorylation of p38 and ERK activation without markedly affecting JNK activation. Therefore, our results indicated that Kaempferol may inhibit IL-1 $\beta$ stimulated inflammation responses by suppressing the MAPK related ERK and P38 pathways.

Kaempferol, a natural flavonoid extracted from various fruits, has exerted its anti-inflammatory properties in various diseases. Previous studies have reported that Kaempferol inhibits the expression of iNOS in activated macrophages and decreases LPS-induced COX-2 level in RAW 264.7 cells $(29,30)$. Kaempferol can also inhibit IL-1 1 -induced the production of MMPs in rheumatoid arthritis synovial fibroblasts (31). Interestingly another in vivo study reported that kaempferol could not inhibit MMP-13 induction in IL-1 $\beta$ treated SW1353 cells at 5-25 $\mu \mathrm{M}$ (32). The SW1353 chondrosarcoma cell line is widely used as a candidate in vitro system for exploring chondrocytes anabolism (33). In contrast, however, we found Kaempferol strikingly inhibited MMP-13 induction in IL-1 $\beta$ treated rat chondrocytes at $10-20 \mu \mathrm{M}$ as well as other MMPs. The cause of the different results may explain to that SW1353 cells may not be a very good alternative for studying chondrocytes anabolism in vitro (34). It was interesting that a recent study reported that kaempferol $(25-100 \mu \mathrm{M})$ could downregulate the IL-1 $\beta$-induced COX2 and iNOS expression via NF- $\kappa B$ pathways in chondrocytes (35). However, in the present study, we observed that kaempferol at $10-20 \mu \mathrm{M}$ concentrations strikingly inhibited Cox 2 and iNOS production as well as other inflammation responses.

The limitation of this study was that we failed to elucidate its protective effects in OA in animal experiments. Further research was required to clarify its anti-inflammation effects in vivo. Besides, in present study our data simply showed the inhibition of Kaempferol on the production of inflammatory mediators was possibly by alleviating of activation of the p38 and ERK signaling pathway, but we failed to conduct more experiments to provide detailed insight about the protective effects of kaempferol in OA. Thus, deeper investigation is necessary to ascertain its intrinsic mechanism.

Collectively,ourstudy provided a new insight into the protective effects of kaempferol in OA. Kaempferol could suppress the IL-1 $\beta$-induced inflammatory reactions such as iNOS2, COX2, MMPs and ADAMTS5. Moreover, cartilage degeneration could also be reversed by kaempferol. Furthermore, Our results also indicate that the downregulation of MMPs with kaempferol treatment was putatively mediated by the MAPK related ERK 
and P38 pathway. These findings indicated that kaempferol may serve as a potent anti-arthritic agent for the treatment of OA.

\section{Acknowledgements}

Not applicable.

\section{Funding}

This research project was supported by the National Natural Science Foundation of China, China (grant no. 81772390) and the Huazhong University of Science and Technology \& Independent Innovation Research Foundation, China (grant no. 2017KFYXJJ104).

\section{Availability of data and materials}

All data generated or analyzed during this study are included in this published article.

\section{Authors' contributions}

HY and XH conceived the idea for the study. XH carried out the research. QP, ZM, PW, RZ, XM and JC analyzed and interpreted the data. HY and $\mathrm{XH}$ wrote the manuscript.

\section{Ethics approval and consent to participate}

Animal experiment procedures were approved by the Animal Care and Use Committee of Tongji Medical College.

\section{Patient consent for publication}

Not applicable.

\section{Competing interests}

The authors declare that they have no competing interests.

\section{References}

1. Wieland HA, Michaelis M, Kirschbaum BJ and Rudolphi KA Osteoarthritis-an untreatable disease? Nat Rev Drug Discov 4 331-344, 2005.

2. Goldring MB and Goldring SR: Articular cartilage and subchondral bone in the pathogenesis of osteoarthritis. Ann N Y Acad Sci 1192: 230-237, 2010.

3. Felson DT: Developments in the clinical understanding of osteoarthritis. Arthritis Res Ther 11: 203, 2009.

4. Wallace IJ, Worthington S, Felson DT, Jurmain RD, Wren KT, Maijanen H, Woods RJ and Lieberman DE: Knee osteoarthritis has doubled in prevalence since the mid-20th century. Proc Natl Acad Sci USA 114: 9332-9336, 2017.

5. Filardo G, Kon E, Longo UG, Madry H, Marchettini $P$, Marmotti A, Van Assche D, Zanon G and Peretti GM: Non-surgical treatments for the management of early osteoarthritis. Knee Surg Sports Traumatol Arthrosc 24: 1775-1785, 2016.

6. Heinegård D and Saxne T: The role of the cartilage matrix in osteoarthritis. Nat Rev Rheumatol 7: 50-56, 2011.

7. Goldring MB, Otero M, Plumb DA, Dragomir C, Favero M, El Hachem K, Hashimoto K, Roach HI, Olivotto E, Borzì RM and Marcu KB: Roles of inflammatory and anabolic cytokines in cartilage metabolism: Signals and multiple effectors converge upon MMP-13 regulation in osteoarthritis. Eur Cell Mater 21: 202-220, 2011
8. Mitchell PG, Magna HA, Reeves LM, Lopresti-Morrow LL, Yocum SA, Rosner PJ, Geoghegan KF and Hambor JE: Cloning, expression, and type II collagenolytic activity of matrix metalloproteinase-13 from human osteoarthritic cartilage. J Clin Invest 97: 761-768, 1996.

9. Kobayashi M, Squires GR, Mousa A, Tanzer M, Zukor DJ, Antoniou J, Feige U and Poole AR: Role of interleukin-1 and tumor necrosis factor alpha in matrix degradation of human osteoarthritic cartilage. Arthritis Rheum 52: 128-135, 2005.

10. Fermor B, Christensen SE, Youn I, Cernanec JM, Davies CM and Weinberg JB: Oxygen, nitric oxide and articular cartilage. Eur Cell Mater 13: 56-65, 2007.

11. Abramson SB, Attur M, Amin AR and Clancy R: Nitric oxide and inflammatory mediators in the perpetuation of osteoarthritis. Curr Rheumatol Rep 3: 535-541, 2001.

12. Henrotin Y, Lambert C, Couchourel D, Ripoll C and Chiotelli E: Nutraceuticals: Do they represent a new era in the management of osteoarthritis?-a narrative review from the lessons taken with five products. Osteoarthritis Cartilage 19: 1-21, 2011.

13. Calderón-Montaño JM, Burgos-Morón E, Pérez-Guerrero C and López-Lázaro M: A review on the dietary flavonoid kaempferol. Mini Rev Med Chem 11: 298-344, 2011.

14. Chen AY and Chen YC: A review of the dietary flavonoid, kaempferol on human health and cancer chemoprevention. Food Chem 138: 2099-2107, 2013.

15. Panahi Y, Alishiri GH, Bayat N, Hosseini SM and Sahebkar A: Efficacy of Elaeagnus Angustifolia extract in the treatment of knee osteoarthritis: A randomized controlled trial. EXCLI J 15: 203-210, 2016.

16. Zhou Y, Zhou Y, Tao H, Li Y, Deng M, He B, Xia S, Zhang C and Liu S: Berberine promotes proliferation of sodium nitroprusside-stimulated rat chondrocytes and osteoarthritic rat cartilage via Wnt//3-catenin pathway. Eur J Pharmacol 789: 109-118, 2016.

17. Livak KJ and Schmittgen TD: Analysis of relative gene expression data using real-time quantitative PCR and the 2(-Delta Delta C(T)) method. Methods 25: 402-408, 2001.

18. Rahmati M, Mobasheri A and Mozafari M: Inflammatory mediators in osteoarthritis: A critical review of the state-of-the-art, current prospects, and future challenges. Bone 85: 81-90, 2016.

19. Dieppe PA and Lohmander LS: Pathogenesis and management of pain in osteoarthritis. Lancet 365: 965-973, 2005.

20. Wojdasiewicz P, Poniatowski $Ł A$ and Szukiewicz D: The role of inflammatory and anti-inflammatory cytokines in the pathogenesis of osteoarthritis. Mediators Inflamm 2014: 561459, 2014.

21. Goldring MB and Otero M: Inflammation in osteoarthritis. Curr Opin Rheumatol 23: 471-478, 2011.

22. Berenbaum F: Osteoarthritis as an inflammatory disease (osteoarthritis is not osteoarthrosis!). Osteoarthritis Cartilage 21: 16-21, 2013.

23. Stöve J, Huch K, Günther KP and Scharf HP: Interleukin-1beta induces different gene expression of stromelysin, aggrecan and tumor-necrosis-factor-stimulated gene 6 in human osteoarthritic chondrocytes in vitro. Pathobiology 68: 144-149, 2000.

24. Gu H, Jiao Y, Yu X, Li X, Wang W, Ding L and Liu L: Resveratrol inhibits the IL-1 $\beta$-induced expression of MMP-13 and IL-6 in human articular chondrocytes via TLR4/MyD88-dependent and -independent signaling cascades. Int J Mol Med 39: 734-740, 2017.

25. Lei M, Wang JG, Xiao DM, Fan M, Wang DP, Xiong JY, Chen Y, Ding Y and Liu SL: Resveratrol inhibits interleukin 1 $\beta$-mediated inducible nitric oxide synthase expression in articular chondrocytes by activating SIRT1 and thereby suppressing nuclear factor- $\kappa$ B activity. Eur J Pharmacol 674: 73-79, 2012.

26. Saklatvala J: Inflammatory signaling in cartilage: MAPK and NF-kappaB pathways in chondrocytes and the use of inhibitors for research into pathogenesis and therapy of osteoarthritis. Curr Drug Targets 8: 305-313, 2007.

27. Zeng L, Rong XF, Li RH and Wu XY: Icariin inhibits MMP-1, MMP-3 and MMP-13 expression through MAPK pathways in IL-1 $\beta$-stimulated SW1353 chondrosarcoma cells. Mol Med Rep 15: 2853-2858, 2017.

28. Jeong JW, Lee HH, Lee KW, Kim KY, Kim SG, Hong SH, Kim GY, Park C, Kim HK, Choi YW and Choi YH: Mori folium inhibits interleukin-1 $\beta$-induced expression of matrix metalloproteinases and inflammatory mediators by suppressing the activation of NF- $\kappa$ B and p38 MAPK in SW1353 human chondrocytes. Int J Mol Med 37: 452-460, 2016. 
29. Kim SK, Kim HJ, Choi SE, Park KH, Choi HK and Lee MW: Anti-oxidative and inhibitory activities on nitric oxide (NO) and prostaglandin E2 (COX-2) production of flavonoids from seeds of Prunus tomentosa Thunberg. Arch Pharm Res 31: 424-428, 2008.

30. Hämäläinen $M$, Nieminen R, Vuorela $P$, Heinonen $M$ and Moilanen E: Anti-inflammatory effects of flavonoids: genistein, kaempferol, quercetin, and daidzein inhibit STAT-1 and NF-kappaB activations, whereas flavone, isorhamnetin, naringenin, and pelargonidin inhibit only NF-kappaB activation along with their inhibitory effect on iNOS expression and NO production in activated macrophages. Mediators Inflamm 2007: 45673, 2007.

31. Yoon HY, Lee EG, Lee H, Cho IJ, Choi YJ, Sung MS, Yoo HG and Yoo WH: Kaempferol inhibits IL-1 $\beta$-induced proliferation of rheumatoid arthritis synovial fibroblasts and the production of COX-2, PGE2 and MMPs. Int J Mol Med 32: 971-977, 2013

32. Lim H, Park H and Kim HP: Effects of flavonoids on matrix metalloproteinase-13 expression of interleukin-1 $\beta$-treated articular chondrocytes and their cellular mechanisms: Inhibition of c-Fos/AP-1 and JAK/STAT signaling pathways. J Pharmacol Sci 116: 221-231, 2011.
33. Chang CC, Hsieh MS, Liao ST, Chen YH, Cheng CW, Huang PT, Lin YF and Chen CH: Hyaluronan regulates PPAR $\gamma$ and inflammatory responses in IL-1 $\beta$-stimulated human chondrosarcoma cells, a model for osteoarthritis. Carbohydr Polym 90: 1168-1175, 2012.

34. Gebauer M, Saas J, Sohler F, Haag J, Söder S, Pieper M, Bartnik E, Beninga J, Zimmer R and Aigner T: Comparison of the chondrosarcoma cell line SW1353 with primary human adult articular chondrocytes with regard to their gene expression profile and reactivity to IL-1beta. Osteoarthritis Cartilage 13: 697-708, 2005.

35. Zhuang Z, Zhuang Z, Ye G and Huang B: Kaempferol alleviates the interleukin-1 $\beta$-induced inflammation in rat osteoarthritis

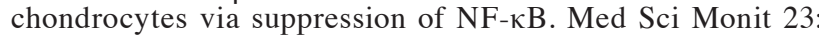
3925-3931, 2017.

(i) $\ominus$ This work is licensed under a Creative Commons

Attribution-NonCommercial-NoDerivatives 4.0 International (CC BY-NC-ND 4.0) License. 